\title{
La relación del logro de la lectura con la lateralidad, el movimiento ocular sacádico y el color en los niños
}

\section{René Tacuri ${ }^{\text {iD }, \text { Marcelo Bernal }}$ iD, Román Buñay}

Facultad de Psicología, Universidad de Cuenca

Autor de Correspondencia: rdavid_90@hotmail.com

Fecha de recepción: 10 de diciembre 2017 - Fecha de aceptación: 5 de febrero 2018

\begin{abstract}
Several authors consider reading an important activity in school, professional and personal life. Perceptual processes, hemispheric lateralization, vision and phonological awareness are factors that intervene in reading skills that can influence the way of learning. This study aimed to evaluate the effect of the use of Irlen color transparencies on the quality of ocular follow-up during reading and the possible relationship with laterality. Participants were fifty-three (53) children, 6 and 7 years old, respectively 32 children with Irlen Syndrome (IS) or visual stress and 21 children as the reference group. All children were subjected to a series of visual standard tests to assess color blindness (Ishihara test), neuropsychological maturity (CUMANES test), Irlen syndrome (IRPS test ${ }^{1}$ ) and rapid eye movement (K-D test ${ }^{2}$ ). Research results reveal that reading capacity is not related to laterality, the use of color significantly improves the perceptual process of reading, and differences in reading attainment is noteworthy between children with Irlen and children without Irlen syndrome. New studies are suggested to consider the effect of attentional abilities, phonological awareness and verbal memory in reading.
\end{abstract}

Keywords: Laterality, saccadic eye movements, Irlen syndrome, reading proficiency, color K-D test.

\section{RESUMEN}

Varios autores consideran a la lectura como una actividad de suma importancia en la vida académica, profesional y personal de un ser humano. Leemos de todo, para nosotros mismos y para todos, sin embargo, los procesos perceptivos, la lateralización hemisférica, la visión y conciencia fonológica son factores intervinientes en la destreza de la lectura que pueden influir en la forma de adquisición del aprendizaje de la misma. El objetivo principal de este estudio fue evaluar el efecto que produce el uso de las láminas de color Irlen ${ }^{\circledR}$ en la calidad del seguimiento ocular durante la lectura y la posible relación con la lateralidad. Los participantes fueron cincuenta y tres (53) escolares de entre 6 y 7 años, 32 con estrés visual y 21 del grupo de referencia, a los cuales se les hizo exámenes visuales estandarizados, pruebas de ceguera al color con el test Ishihara, la madurez neuropsicológica a través del test CUMANES, síndrome Irlen o estrés visual con EPLI ${ }^{1}$ y los movimientos oculares con la prueba $\mathrm{K}-\mathrm{D}^{2}$. El análisis estadístico muestra que la lateralidad no está relacionada con la capacidad lectora; existe mejorías significativas en el proceso perceptivo para la lectura con el uso del color; sin embargo, no se encontraron valores representativos para la comprensión y número de palabras por minuto. Hay diferencias significativas en la capacidad lectora entre niños con Irlen y niños sin Irlen, y se sugiere nuevos estudios que consideren habilidades atencionales, conciencia fonológica y memoria verbal en la lectura.

Palabras clave: Lateralidad, movimientos oculares, habilidad de lectura; síndrome Irlen; color; prueba K-D.

\footnotetext{
${ }^{1}$ Irlen Reading Perceptual Scale test / Escala Perceptual de Lectura Irlen

${ }^{2}$ King-Devick test / prueba King-Devick
} 


\section{INTRODUCCIÓN}

El aprendizaje de la lectura es la etapa crucial de inicio de la escolaridad del niño, un buen proceso lector le permitiría adquirir conocimientos que estén acorde a la edad de desarrollo. Sin embargo, no todos los escolares gozan del privilegio de aprender a leer y de leer para aprender, dos procesos importantes que se dan especialmente entre los 5 a 9 años, es decir entre los segundos y terceros grados de educación general básica. Esta etapa está marcada por la consolidación del aprendizaje de la lectura o la aparición de un sin número de problemas de aprendizaje entre los cuales se destaca la presencia de la dislexia. La dislexia o trastorno de la lectura puede afectar el desarrollo armónico de un 5 a $15 \%$ de escolares, la misma que no necesariamente depende de una deficiencia intelectual, emocional o motivacional, aunque en casos severos frecuentemente pueden estar asociados a otros trastornos específicos del desarrollo (Caicedo, 2012; Toro \& Yepes, 2004).

Mesonero (1994, citado en Mayolas, Aparicio, Masia, Pi, Aparicio, \& Masia, 2010), relaciona los problemas de aprendizaje de la lectura, posiblemente entre otras cosas, a las dificultades de discriminación entre derecha e izquierda, retraso de maduración nerviosa y a las alteraciones de la lateralidad. Barrero, Vergara, \& Martín-Lobo (2015) también señalan que, aunque la lateralidad no necesariamente pueda determinar problemas de aprendizaje, muy probablemente ésta puede ser la causa de un aprendizaje inadecuado. El manual de diagnóstico DSM-V (APA, 2013) cita a la dislexia como uno de los trastornos específicos del aprendizaje en donde están afectados la precisión en la lectura de las palabras, velocidad, fluidez y comprensión lectora. Rosselli, Matute, \& Ardila (2006) asocian la velocidad lectora con habilidades atencionales y la comprensión con la memoria verbal. Por otra parte, en la búsqueda de justificar el origen y el desarrollo de la dislexia, se han planteado cinco teorías que asocian las dificultades de lectura con factores perceptuales, de asociación y/o funcionalidad cerebral, entre ellas se destacan: i) teoría fonológica, ii) teoría de procesamiento rápido auditivo, iii) teoría visual, iv) teoría cerebelosa y v) la teoría magnocelular (Artigas-Pallarés, 2009; Shaywitz \& Shaywitz, 2005). Cabe señalar que todas estas teorías coinciden estar asociadas a un déficit fonológico (Ramus et al., 2003).

Desde un abordaje perceptual visual, la eficiencia de la lectura está directamente influenciada por la calidad de los movimientos sacádicos, el mismo que podría verse afectado por una alteración del sistema magnocelular ocasionando interferencias en la percepción del texto nuevo (López-Escribano, 2007). Bernal (2015a), Nandakumar \& Leat (2008) e Irlen (2005) coinciden que posiblemente los problemas de lectura pueden deberse a un problema visual, en donde los niños pueden presentar malestares, reconocido como síndrome de sensibilidad escotópica, estrés visual o síndrome de Irlen. En esta misma línea de apreciación, también se ha obtenido resultados positivos en la fluidez y comprensión lectora a través del entrenamiento de los movimientos oculares (Dodick et al., 2017; Leong et al., 2014). Algunos autores postulan que la calidad de la visión para la lectura puede ser mejorada a través de la intervención del color mediante el uso de láminas o lentes coloreados (Bernal, 2015a; Chouinard, Zhou, Hrybouski, Kim, \& Cummine, 2012; Irlen, 2005; López-Escribano, 2007). Chase, Ashourzadeh, Kelly, Monfette, \& Kinsey (2003) realizaron estudios a través de cuatro métodos experimentales incluido el método Irlen, en donde usando láminas de color observaron mejoría en el rendimiento de los participantes en un $25 \%$ en la cantidad de errores y un $8 \%$ en la capacidad de comprensión.

El énfasis de la presente investigación se enfocó en la atención a las teorías que consideran la alteración de la percepción visual (teoría magnocelular y teoría visual) como causantes de los problemas en la lectura. Relacionado con esto, el objetivo principal de este estudio fue evaluar el efecto que produce el uso de las láminas de color Irlen ${ }^{\circledR}$ en la calidad del seguimiento ocular durante la lectura y la posible relación con la lateralidad. 


\section{MATERIALES Y MÉTODOS}

De acuerdo a las características de la muestra y objetivos planteados, esta investigación es de tipo cuasi experimental, cuantitativa. Todos los participantes fueron voluntarios cuyos padres de familia y representantes legales firmaron un consentimiento y acordaron participar. La muestra incluyó a 53 niños y niñas que cursaban el tercero y cuarto grado de educación básica de una escuela pública, de los cuales 32 presentaban Síndrome Irlen (Grupo Irlen), y 21 sin Irlen (Grupo No Irlen) al cual se le refiere como grupo de referencia o comparación. El diagnóstico de Irlen fue realizado por un profesional "Irlen Screener" mediante la utilización de la Escala Perceptual de Lectura Irlen (Bernal, 2015b) y el uso de un set de 10 acetatos de color Irlen. Los criterios de exclusión para la muestra fueron: niños que necesitaban lentes, pero no los usaban durante las aplicaciones de las pruebas, niños que presentaban daño cerebral leve, moderado o profundo, los que presentaban diagnósticos neuropsicológicos y los que necesitaban de currículo diferencial.

Los instrumentos utilizados en la investigación siguieron una secuencia de aplicación estructurada. En primer lugar, los participantes fueron sometidos a una evaluación oftalmológica y una prueba de ceguera al color, posterior a la cual se obtuvieron resultados referentes a problemas visuales existentes, que fueron diagnosticados por el profesional a cargo de estas evaluaciones. Esto motivaría a los participantes a corregir sus problemas visuales para continuar con el siguiente paso que consistió en la aplicación del Cuestionario de Madurez Neuropsicológica "CUMANES" (Portellano, Mateos, \& Martinez Arias, 2012) al total de la muestra con la intención de verificar el índice de desarrollo neuropsicológico en los participantes y adicionalmente obtener información referente a la lateralidad. A continuación se aplicó la prueba King-Devick (Galetta et al., 2011), con el fín de determinar la calidad de los movimientos oculares, tanto en el tiempo y cantidad de errores cometidos. La siguiente evaluación fue la prueba EPLI, misma que tuvo que ser aplicada por profesionales calificados y autorizados. Seguidamente, se procedió a la administración de las pruebas de lectura, adoptando extractos de lectura de 100 palabras de los libros del Ministerio de Educación del Ecuador, que se utilizan en los terceros y cuartos grados de educación general básica.

Para evaluar el nivel de comprensión se incluyeron dos cuentos breves, se registró el tiempo, cantidad de errores y las respuestas a cinco preguntas. El participante leyó el primer cuento luego del cuestionario hipotético de dificultades y malestares -17 preguntas de la sección 2 de la EPLI para las dos categorías-, y el segundo cuento se usó si el evaluador consideraba que existían dificultades en la lectura. Posterior a esta prueba, se realizó una nueva aplicación de la prueba K-D, con la diferencia de que en esta ocasión se utilizó una lámina de color que fue seleccionada por el participante en la prueba diagnóstica.

Cada una de las pruebas fueron llevadas a cabo en los espacios de la escuela participante y las mismas fueron realizadas con cada uno de los participantes, teniendo en cuenta que los espacios sean los más adecuados posible, al igual que los tiempos estándares de evaluación. El intervalo temporal de aplicación de cada prueba fue de al menos un día para evitar la fatiga de los participantes y el aprendizaje de las respuestas en el caso de la prueba K-D.

Para el análisis de resultados se utilizó el programa SPSS versión 21 para las pruebas de normalidad, correlación y comparación según el comportamiento de cada variable estudiada.

\section{RESULTADOS Y DISCUSIONES}

La edad media de la población de estudio fue de 6.96 y la desviación estándar de 0.192, distribuyéndose según la lateralidad como muestra la Tabla 1 . La prueba K-D aplicada a las variables tiempo con y sin color y errores con y sin color, muestra una normalidad en su comportamiento en las primeras, esto ocasiona el uso de las pruebas no paramétricas para el análisis estadístico. 
Tabla 1. Descripción de la población de estudio.

\begin{tabular}{|c|c|c|c|c|c|c|c|c|c|c|}
\hline \multirow{3}{*}{$\begin{array}{l}\text { Genero } \\
\text { Edad } \\
\text { Variable }\end{array}$} & \multicolumn{5}{|c|}{ Hombre } & \multicolumn{4}{|c|}{ Mujer } & \multirow[b]{3}{*}{ Total } \\
\hline & \multicolumn{2}{|c|}{6} & \multicolumn{2}{|c|}{7} & & \multicolumn{2}{|c|}{6} & \multicolumn{2}{|c|}{7} & \\
\hline & LD & LI & LD & LI & Total & LD & LI & LD & LI & \\
\hline Irlen & 0 & 0 & 6 & 5 & 11 & 0 & 0 & 10 & 11 & 21 \\
\hline No Irlen & 0 & 2 & 6 & 5 & 13 & 0 & 0 & 5 & 3 & 8 \\
\hline Total & & & & & 24 & & & & & 29 \\
\hline
\end{tabular}

Variable: LD: Lateralidad definida, LI: Lateralidad indefinida (Tacuri, 2017)

En una comparación entre el grupo Irlen y el grupo no Irlen se demuestra que existen diferencias significativas en la capacidad lectora $p<0.05$, sin embargo, no existen diferencias estadísticamente reveladoras en la prueba K-D $p>0.05$ (ver Tabla 2). El grupo no Irlen muestra un mejor rendimiento en la capacidad lectora, tanto en la cantidad de errores, mejora de nivel de comprensión y disminución del tiempo de lectura. Estas diferencias significativas en la capacidad lectora entre las poblaciones que no tienen SI o estrés visual y quienes, si presentan este síndrome, sugieren que el factor perceptual está implicado en la capacidad lectora.

Tabla 2. Comparación de lectura y movimientos sacádicos según los grupos Irlen y No Irlen.

\begin{tabular}{|c|c|c|c|c|c|c|c|}
\hline & & & \multicolumn{3}{|c|}{ Lectura } & \multicolumn{2}{|c|}{ Prueba K-D } \\
\hline & & & Errores & Comprensión & Tiempo & Errores & Tiempo \\
\hline \multirow{3}{*}{ Grupo } & Irlen & Media & 8 & 3.9 & 111.81 & 6.22 & 98.66 \\
\hline & No Irlen & Media & 3 & 3.95 & 89.05 & 7.67 & 93.62 \\
\hline & & Sig. & 0.001 & 0.033 & 0.026 & 0.647 & 0.512 \\
\hline
\end{tabular}

Sig.: nivel de significancia (Tacuri, 2017)

Tabla 3. Chi Cuadrado $\left(\chi^{2}\right)$ entre lateralidad, comprensión lectora y normalidad de la prueba K-D (Fuente: Tacuri, 2017).

\begin{tabular}{|c|c|c|c|c|c|}
\hline \multirow{3}{*}{ Variable } & \multirow{3}{*}{ Condición } & \multicolumn{4}{|c|}{ Análisis Chi-Cuadrado } \\
\hline & & \multicolumn{2}{|c|}{ Comprensión lectora } & \multicolumn{2}{|c|}{ Normalidad K-D } \\
\hline & & $\chi^{2}$ & $p$ & $\chi^{2}$ & $p$ \\
\hline \multirow{2}{*}{ Lateralidad } & sin color & 5.852 & 0.321 & 1.535 & 0.215 \\
\hline & con color & 4.261 & 0.372 & 2.133 & 0.333 \\
\hline
\end{tabular}

$\mathrm{Al}$ aplicar la prueba $\chi^{2}$, con relación a la variable lateralidad, comprensión lectora y normalidad de movimientos oculares (normalidad prueba K-D) tanto en el grupo de comparación y el experimental, no se encontraron relaciones estadísticamente reveladoras Tabla 3). Se analizó la diferencia entre dos grupos según la definición de la lateralidad mediante la prueba U de Mann-Whitney. Los resultados en la Tabla 4 muestran que no existe diferencia importante entre ambos grupos. Por lo que se puede afirmar que la lateralidad no es una variable que produzca efecto sobre los errores y el tiempo de lectura.

Tabla 4. Prueba U de Mann-Withney entre Lateralidad y Lectura (errores y tiempo) (Fuente: Tacuri, 2017).

\begin{tabular}{cccccc}
\hline \multirow{2}{*}{ Variable } & \multirow{2}{*}{ Condición } & \multicolumn{2}{c}{ Errores } & \multicolumn{2}{c}{ Tiempo } \\
\cline { 3 - 6 } & & $\mathrm{U}$ de Mann & $p$ & $\mathrm{U}$ de Mann & $p$ \\
\hline \multirow{2}{*}{ Lateralidad } & sin color & 318.50 & .561 & 312.00 & .488 \\
& con color & 126.00 & .939 & 118.00 & .706 \\
\hline
\end{tabular}

De acuerdo a estos resultados, podemos indicar que la lateralidad no es un factor influyente ni en la lectura ni en la capacidad de movimientos oculares, pues tanto los participantes que tienen lateralidad 
definida y lateralidad indefinida, presentan similares comportamientos, sin cambios significativos. Estos resultados se enmarcan con las afirmaciones de Barrero et al. (2015), Mayolas et al. (2010) y Portellano (2004) quienes señalan que la lateralidad no tiene relación con la capacidad lectora, aunque en problemas de aprendizaje puedan aparecer problemas de lateralidad. Sobre el efecto que produce el uso de las láminas de color, puede verse que existen únicamente diferencias reveladoras en los errores en la lectura $(p=0.002)$ y el tiempo en los movimientos oculares $(p=0.005)$. Sin embargo, aunque existan diferencias entre las medias de comprensión y tiempo de la lectura y la variable errores en la prueba K$\mathrm{D}$, estos cambios no son realmente substanciales $p>0.05$.

La cantidad de errores cometidos en la lectura se ve beneficiada con el uso del color con una media de 6 sin color y una media de 5.50 con color (Fig. 1, izquierda), mientras que el tiempo empleado en la prueba K-D también mejora por su disminución de una media de 96.66 a 93.41 (Fig. 1, derecha). Los colores de mayor preferencia escogidos por la muestra participante fueron el "aqua" con el 20\%; "purple" 13\%; "green" y "turquoise" $10 \%$, y hubo también preferencias únicas por el uso de otros colores o la combinación de dos o más. Estos resultados muestran que existe una mejoría a través del uso del color en la disminución de errores y del tiempo en la prueba de movimientos oculares. A pesar de que las medias de las variables comprensión lectora, tiempo lector, y errores en los movimientos sacádicos mejoran, estos cambios no son estadísticamente sustanciales.

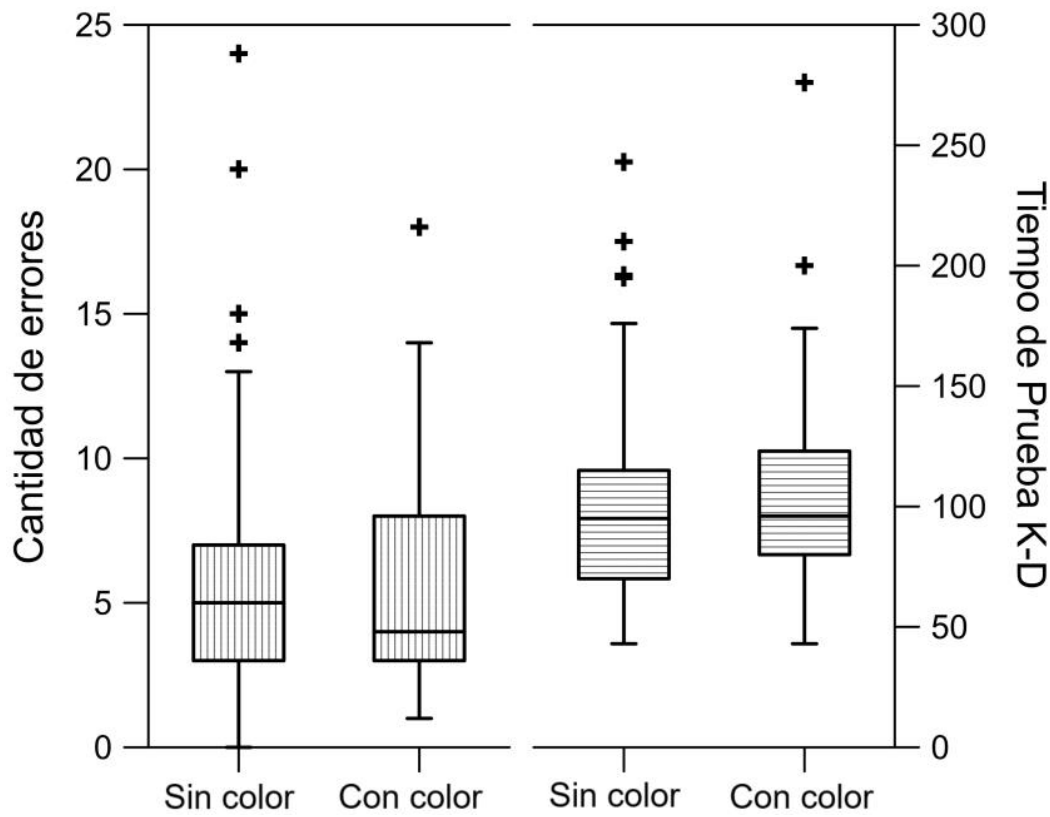

Figura 1. Izquierda: diagrama de Box-Whisker de las diferencias significativas en la cantidad de errores cometidos con y sin color $(p=0.002)$ y Derecho: diagrama de Box-Whisker de las diferencias significativas en el tiempo de ejecución de la prueba K-D $(p=0.005)$.

López-Escribano (2007) afirma que el sistema magnocelular podría afectar la percepción del texto y el uso de lentes de color podría ser una forma de corrección a este problema, sin embargo Chase et al. (2003) en sus modelos experimentales señalan que existen mejorías significativas únicamente con el uso de láminas de color, mientras que al usar lentes coloreados no mostraron cambios importantes.

\section{CONCLUSIONES}

Es de conocimiento general que la adquisición de la lectura es un proceso complejo y que causa una serie de situaciones adversas en el rendimiento escolar. Se tiene noción también que en el proceso de aprendizaje de la lectura intervienen un sin número de variables que van desde el medio ambiente 
familiar, socioeconómico, físico y biológico de las personas hasta situaciones muy particulares para cada individuo. Dentro de algunas de las variables que intervienen en la adquisición de la lectura están los movimientos oculares y la lateralidad y su relación con el uso de transparencias de color, variables que no han sido estudiadas dentro de nuestros contextos educativos ecuatorianos. El objetivo principal de este estudio fue evaluar el efecto que produce el uso de las láminas de color Irlen ${ }^{\circledR}$ en la calidad del seguimiento ocular durante la lectura y la posible relación con la lateralidad con el fin de contribuir con una pieza a ese rompecabezas que significa el proceso de lectura y presentar posibles soluciones a los problemas de lectura. La lateralidad es una variable que no se encuentra relacionada con alguna característica de los movimientos oculares o el proceso lector, es decir la lateralidad no afecta la calidad de la lectura, sin embargo, para investigaciones futuras, puede ser conveniente un análisis con casos con diagnóstico de dislexia.

Existen diferencias significativas de la capacidad lectora entre niños sin Síndrome Irlen y niños con Síndrome Irlen, caracterizando una deficiente calidad para leer en aquellos que presentan Síndrome Irlen o estrés visual. Sin embargo los planteamientos de Chase et al. (2003) e Irlen (2005) sobre uso de las transparencias de color para el beneficio en la lectura, tampoco son significativos en todas las variables estudiadas. Una de las hipótesis posibles del por qué no existe mejoría, podría ser una asociación con otras habilidades de tipo atencionales, la memoria verbal (Rosselli et al., 2006) o el hábito lector. Es conveniente a futuro analizar cuáles son las soluciones más efectivas para contrarrestar o disminuir el impacto del Síndrome Irlen o estrés visual sobre la lectura y hacer un énfasis especial en las habilidades neuropsicológicas como la atención, memoria verbal y conciencia fonológica.

El enfoque de la teoría visual y magnocelular de este estudio, detalla que existe dificultad significativa para leer, por lo tanto, se recomienda que los centros educativos con el respaldo y apoyo de padres de familia o representantes legales de los niños, no descuiden una valoración del proceso perceptivo en edades tempranas puesto que se podría evitar problemas mayores en función de la definición de la conciencia fonológica, el reconocimiento simbólico acorde a su edad de desarrollo y demás factores que afectan directa o indirectamente en la adquisición de las habilidades lectoras.

Además de que el aprendizaje de la lectura sea un proceso continuo, es vital que tanto los centros educativos y las familias estimulen el hábito lector mediante actividades que sean llamativas y estimulantes para los niños, orientando a que el niño pueda encontrar agrado por leer y más no un rechazo por la obligatoriedad y repetitividad (Montalvo, 2014).

\section{AGRADECIMIENTOS}

En primer lugar, expresamos nuestro agradecimiento al equipo de investigación del proyecto "Efectos del Uso de Transparencias de Color en la Fluidez y Comprensión Lectora en Escolares con Síndrome Irlen". Igualmente, nuestro sincero agradecimiento a la administración de la Escuela Manuela Cañizares, a su personal docente, padres de familia y representantes legales de los niños participantes por su apertura a la investigación. Finalmente, nuestro reconocimiento y agradecimiento sincero a la Dirección de Investigación de la Universidad de Cuenca por el apoyo académico y financiero, ya que, sin este aporte importante, esta investigación no hubiese podido ejecutarse.

\section{REFERENCIAS}

Artigas-Pallarés, J. (2009). Dyslexia: a disease, a disorder or something else? Revista de Neurología, 48(Suppl 2), S63-9.

American Psychiatric Association. (2013). Diagnostic and statistical manual of mental disorders: DSM-5 ( $5^{\text {th }}$ ed.). Arlington, VA: American Psychiatric Association.

Barrero, M., Vergara, E., Martín-Lobo, M. P. (2015). Avances neuropsicológicos para el aprendizaje matemático en educación infantil: la importancia de la lateralidad y los patrones básicos del 
movimiento. Edma 0-6: Educación Matemática en la Infancia, ISSN-e 2254-8351, 4(2), 22-31.

Bernal, M. (2015a). Dislexia, déficit de atención/hiperactividad o Síndrome Irlen. Maskana, 6(2), 109-119.

Bernal, M. (2015b). Prevalencia del síndrome Meares-Irlen/Estrés Visual que afecta la lectura en niños de tercer grado. Maskana, 6(1), 19-25.

Caicedo, H. (2012). Neuroaprendizaje: Una propuesta educativa (2 ${ }^{\mathrm{a}}$ ed.). Bogota D.C., Colombia: Ediciones de la U, $206 \mathrm{p}$.

Chase, C., Ashourzadeh, A., Kelly, C., Monfette, S., Kinsey, K. (2003). Can the magnocellular pathway read? Evidence from studies of color. Vision Research, 43(10), 1211-1222. https://doi.org/10.1016/S0042-6989(03)00085-3

Chouinard, B. D., Zhou, C. I., Hrybouski, S., Kim, E. S., Cummine, J. (2012). A functional neuroimaging case study of Meares-Irlen syndrome/visual stress (MISViS). Brain Topography, 25(3), 293-307. https://doi.org/10.1007/s10548-011-0212-z

Dodick, D., Starling, A. J., Wethe, J., Pang, Y., Messner, L. V, Smith, C., Master, C. L., HalkerSingh, R. B., Vargas, B. B., Bogle, J. M., Mandrekar, J., Talabar, A., Leong, D. (2017). The effect of in school saccadic training on reading fluency and comprehension in first and second grade students: A randomized controlled trial. Journal of child neurology, 32(1), 104-111. https://doi.org/10.1177/0883073816668704

Galetta, K. M., Brandes, L. E., Maki, K., Dziemianowicz, M. S., Laudano, E., Allen, M., Lawler, K., Sennett, B., Wiebe, D., Devick, S., Messner, L. V., Galetta, S. L., Balcer, L. J. (2011). The KingDevick test and sports-related concussion: Study of a rapid visual screening tool in a collegiate cohort. Journal of the Neurological Sciences, 309(1-2), 34-39. https://doi.org/10.1016/j.jns.2011.07.039

Irlen, H. (2005) Reading by the colors: Overcoming dyslexia and other reading disabilities through the Irlen method. A Perigee Book. New York, USA: Penguin Group (USA) Inc., 211 p.

Leong, D. F., Master, C. L., Messner, L. V, Pang, Y., Smith, C., Starling, A. J. (2014). The Effect of Saccadic Training on Early Reading Fluency. Clinical pediatrics, 53(9), 858-64. https://doi.org/10.1177/0009922814532520

López-Escribano, C. (2007). Contributions of neuroscience to the diagnosis and educational treatment of developmental dyslexia . Revista de Neurología, 44(3), 173-180.

Mayolas, C., Aparicio, V., Masia, J. J. R., Pi, M., Aparicio, A., Masia, J. J. R. (2010). Relación entre la lateralidad y los aprendizajes escolares. Apunts. Educació Física i, 101(1577-4015), 32-42.

Montalvo, J. (2014). Estimulación de los centros cerebrales del habla y el lenguaje en adquisición de lectoescritura en niños de 4 a 6 años. Maskana, 5(2), 29-40.

Nandakumar, K., Leat, S. J. (2008). Dyslexia: A review of two theories. Clinical and Experimental Optometry, 91(4), 333-40. doi: 10.1111/j.1444-0938.2008.00277.x

Portellano, J. A. (2004). La dislexia: en todas las edades. A distancia, 2, 81-85.

Portellano, J. A., Mateos, R., Martinez Arias, R. (2012). CUMANES Cuestionario de madurez neuropsicológica escolar. España: TEA Ediciones, $30 \mathrm{p}$.

Ramus, F., Rosen, S., Dakin, S. C., Day, B. L., Castellote, J. M., White, S., Frith, U. (2003). Theories of developmental dyslexia: Insights from a multiple case study of dyslexic adults. Brain, 126(4), 841-865. https://doi.org/10.1093/brain/awg076

Rosselli, M., Matute, E., Ardila, A. (2006). Neuropsychological predictors of reading ability in Spanish. Revista de Neurología, 42(4), 202-210.

Shaywitz, S. E., Shaywitz, B. A. (2005). Dyslexia (Specific Reading Disability). Biological Psychiatry, 57(11), 1301-1309. https://doi.org/10.1016/j.biopsych.2005.01.043

Tacuri, R. D. (2017). La lateralidad y los efectos del color sobre la lectura y los movimientos sacádicos. La Rioja, España: Universidad Nacional de La Rioja.

Toro, R. J., Yepes, L. E. (2004). Fundamentos de Medicina, Psiquiatria. (J. E. Téllez-Vargas, Ed.) (4. ${ }^{a}$ ed.). Medellin, Colombia: CIB (Corporacion para Investigaciones Biologicas)., $463 \mathrm{p}$. 